\title{
Immunotherapy for renal cancer: Sequencing and combinations
}

Grant D. Stewart ${ }^{1}$, Maria De Santis², Bernard Escudier ${ }^{3}$, Thomas Powles ${ }^{4}$, Guru Sonpavde ${ }^{5}$

${ }^{1}$ Academic Urology Group, University of Cambridge, Addenbrooke's Hospital, Cambridge, UK; ${ }^{2}$ Cancer Research Centre, University of Warwick, UK; ${ }^{3}$ Gustave Roussy, Villejuif, France; ${ }^{4}$ Bart's Cancer Institute, London, UK; ${ }^{5}$ Department of Medicine, Section of Hematology-Oncology, University of Alabama at Birmingham (UAB), Birmingham, AL, USA

\section{Corresponding Author}

Guru Sonpavde, MD

UAB Comprehensive Cancer Center, 1720 2nd Ave. S., NP2540B

Birmingham, AL 35294

E-mail: gsonpavde@uabmc.edu

Phone: 205-975-2914, Fax: 205-975-3910

Number of words in abstract: $287 / 300$

Number of words in text: $2612 / 4000$

Number of references: $47 / 50$

Number of figures: 2

Number of tables: 1

\section{Disclosures}

- Grant D Stewart: Educational grants: Pfizer; Speaker fees: Pfizer; Consultancy: Cambridge Medical Robotics

- Maria De Santis: Consultancy: GlaxoSmithKline, Janssen, Bayer, Novartis, Pierre Fabre, Astellas, Amgen, Eisai Inc., ESSA, Merck, and Synthon; speaker fees: Pfizer, Takeda, Sanofi Aventis, Shionogi, Celgene, and Teva OncoGenex; participated in trials: Pierre Fabre, Astellas, Exelixis, Bayer, and Roche; has received fellowship and travel grants: Bayer, Novartis, Ferring, Astellas, Sanofi Aventis, and Janssen; Research Grants: Pierre Fabre; honoraria: AstraZeneca; and is associated with Amgen.

- Bernard Escudier: Honoraria from Bayer, Novartis, Pfizer, Bristol-Myers Squibb, and Exelixis.

- Tom Powles: Consultancy: Novartis, Pfizer, and GlaxoSmithKline; Speaker fees: Novartis, Pfizer, GlaxoSmithKline, and Genentech; Participated in trials for GlaxoSmithKline, Pfizer, BMS, Genentech, and Genetech; Educational grants: GlaxoSmithKline, Pfizer, and Novartis.

- Guru Sonpavde: Educational grants: Boehringer-Ingelheim, Bayer, Onyx-Amgen, Merck; Consultancy: Pfizer, Genentech, Novartis, Argos, Merck, Sanofi, Agensys, Clinical Care Options, Astrazeneca, Uptodate, Biotheranostics, Exelixis, BristolMyers-Squibb, Janssen, Amgen, Eisai, personal fees from NCCN (National Comprehensive Cancer Network). 


\section{Abstract}

Context: Current therapy for renal cell carcinoma $(R C C)$ generally consists of the sequential administration of single agent therapy. Given the advent of T-cell checkpoint inhibitors, the role of combinations including these agents is being intensely interrogated.

Objective: To evaluate ongoing trials of combinations including immunotherapy and sequencing of agents to treat RCC.

Evidence Acquisition: Recent data and ongoing trials were analysed to evaluate the direction of research in this arena.

Evidence Synthesis: The favourable therapeutic index of PD-1/PD-L1 inhibitors enables combinations of these agents. Multiple ongoing phase III trials are evaluating the first-line therapy of RCC using a combination of PD-1/PD-L1 inhibitors with VEGF inhibitors or CTLA4 inhibitors. The role of sequencing using single agent sunitinib and avelumab will be evaluated in a randomized phase II trial. The role of vaccine therapy remains unproven. The role of predictive biomarkers to select appropriate therapy requires a larger focus, given the multitude of possible therapies.

Conclusion: Therapy for RCC should be tailored based on both patient and tumour characteristics. Combination therapy and sequencing of single agents may both play roles and are undergoing clinical trial evaluation.

Patient Summary: Combinations of immunotherapy with angiogenesis inhibitors is undergoing vigorous clinical trial evaluation. Sequencing of immunotherapy and antiangiogenic therapy is also undergoing investigation. Clinical trial participation is critically important to develop new drugs and combinations, and biomarkers to select therapy.

\section{Take home message}

The thrust of ongoing clinical research is to combine PD-1 and PD-L1 inhibitors with either anti-angiogenic agents or with CTLA-4 inhibitors as first-line therapy for metastatic renal cell carcinoma $(\mathrm{RCC})$. Sequencing of more tolerable single agent therapy may continue to play a 
role in selected patients ineligible for combination therapy. Additionally, the role of candidate predictive biomarkers requires validation.

Keywords: Renal cell carcinoma; Immunotherapy; Combination; Sequencing 


\section{Introduction}

Renal cell carcinoma (RCC) is exquisitely resistant to chemotherapy. The chemoresistance may be partly attributable to the disease being derived from proximal tubules, which express large amounts of the multidrug resistant P-glycoprotein. Equally, relative to other malignancies, RCC has been particularly sensitive to immunotherapy. The first indication that RCC might be a good target for immunotherapy came from the observation that patients with metastatic RCC occasionally experienced spontaneous regressions after surgical removal of the primary tumour [1-3]. It is also well established that there is profuse inflammatory infiltrate in RCC, although the precise role of each infiltrating cell type (T-cells, natural killer cells, dendritic cells and macrophages) is not established [4]. Until 2005, the median survival of patients with metastatic RCC (mRCC) was approximately 1 year and the only treatments available were interleukin (IL)-2 and interferon (IFN)- $\alpha$ which conferred modest benefits [5]. A major step-forward started in 2005, when new targeted drugs including mammalian target or rapamycin (mTOR) inhibitors (everolimus, temsirolimus), vascular endothelial growth factor (VEGF) inhibitors (sunitinib, sorafenib, axitinib, pazopanib, cabozantinib) and a programmed death (PD)-1 inhibitor (nivolumab) were approved [6,7]. Additionally, the combination of a VEGF inhibitor plus cytokine (bevacizumab plus IFN- $\alpha$ ) and VEGF inhibitor plus mTOR inhibitor (lenvatinib plus everolimus) have demonstrated improved outcomes in the first-line and post-VEGF inhibitor settings respectively, and are approved. Thus, the treatment algorithm includes the use of sunitinib, pazopanib, bevacizumab plus IFN- $\alpha$ or temsirolimus as first-line therapy, and nivolumab, cabozantinib, axitinib or lenvatinib plus everolimus as second-line therapy. Collectively, these agents have extended the median survival of mRCC patients to 2-2.5 years. High dose IL-2 is reserved as first-line therapy for well selected younger patients without comorbidities.

Since 2015 , there has been a further paradigm shift in the management of mRCC with the addition of nivolumab, a T-cell checkpoint inhibitor, to the therapeutic armamentarium for post-VEGF inhibitor patients. The next generation of immunotherapeutics has been 
established to be of benefit in mRCC. Harnessing the immune system has long been of interest because of the potential for durable responses, initially seen with cytokine treatment [8]. The step change in mechanism of action that T-cell checkpoint inhibitors provide is that of immunoediting i.e. altering the balance between the tumour and the immune system [9]. In the elimination phase CD8+ T-cells and natural killer cells destroy a proportion of malignant cells, the surviving cancer cells survive in a constrained state in the presence of immune cells in the equilibrium phase, before entering the escape phase where cancer cells evade immune cell recognition [9]. T-cell checkpoint inhibitors such as PD-1 inhibitors can alter this balance by unleashing the anti-tumour activity of T-cells in the tumour microenvironment [10].

It remains the case that most patients will not show a major durable response to single line immune therapy [11]. As such, combination and sequential therapies are being evaluated. Vanneman and Dranoff have described different potential mechanisms by which combination therapy strategies may work [12]: (i) enhance antigen presentation and T-cell priming; (ii) augment differentiation of memory T cells; (iii) improve antitumour T-cell function; (iv) enhance cytotoxic T lymphocyte (CTL)-mediated lysis of tumour cells; (v) reduce tumour-associated immunosuppression; and (vi) decrease immunosuppressive cell populations.

In view of the increasing number of drugs with differing mechanisms of action in the arsenal of the oncologist, the next questions being answered by clinical trials are the role of sequential or combinatorial therapy with immunotherapeutic agents. Ongoing trials are evaluating the combination of PD-1/PD-ligand (L)-1 inhibitors with either VEGF inhibitors or cytotoxic T-Lymphocyte Antigen (CTLA)-4 inhibitors as first-line therapy (Table 1). In this review, we will summarize this literature and plot a future course.

\section{Evidence acquisition}




\section{$\underline{\text { Search criteria }}$}

A literature search was performed using Pubmed (January 1976-March 2017).

\section{$\underline{\text { The Past: cytokines and historically evaluated combinations }}$}

Interferon-alpha (IFN- $\alpha$ ) and high-dose (HD) IL-2) are the two cytokine therapies which have been proven to be efficacious in mRCC treatment [13]. The exact mechanism by which these agents work is unclear. However, IL-2 is known to stimulate T-cell proliferation and differentiation and IFN- $\alpha$ is antiangiogenic as well as a promoter of antigen presentation and dendritic cell development [4]. IL-2 is a toxic regimen, requiring inpatient administration and resulting in up to $3 \%$ patients dying from treatment; however, there is a $7 \%$ complete response $(\mathrm{CR})$ rate, most of them being durable and potential cures, and around $15 \%$ patients have an objective response [14]. Unsurprisingly, toxicity has limited the use of this agent.

In view of the potential for durable CR in patients treated with HD IL-2, predictive clinical and molecular markers of response have been evaluated in an effort to target this very toxic treatment to the right patients. Several retrospective studies determined that high carbonic anhydrase 9 (CA9) levels as measured by immunohistochemistry could predict which patients would respond to HD IL-2 $[15,16]$. These studies led to the prospective SELECT trial [17] which assessed the predictive ability of favourable histology [18] (alveolar features and no granular or papillary features) and high CA9 levels in 120 patients. Combined pathological features and CA9 levels was not proven to predict response to HD IL-2 [17]. However, in a post-hoc analysis, combined single nucleotide polymorphisms (SNPs) in the Fc-gamma receptors (FCGRs): FCGR2A, FCGR3A, and FCGR2 was associated with outcome following HD IL-2 therapy [19]. 
Combination cytokine and VEGF inhibitor therapies have been evaluated, with bevacizumab (a monoclonal antibody targeting VEGF) being approved for use as first-line therapy in combination with IFN- $\alpha[20,21]$. Bevacizumab has also been combined with HD IL-2 and was found to be feasible, but did not appear to enhance durable responses over historical rates observed with IL-2 alone [22]. However, VEGF tyrosine kinase inhibitor (TKI) plus cytokine and VEGF inhibitor plus mTOR inhibitor combinations have historically demonstrated prohibitive toxicities and/or not resulted in additional long term responses compared with single agent cytokines [23-26]. In contrast to the lack of feasibility of these combinations of older VEGF inhibitors with mTOR inhibitors, the combination of lenvatinib (a VEGF and fibroblast growth factor receptor inhibitor) and everolimus was associated with a significantly longer median progression-free survival (PFS) of 14.6 months compared to 5.5 months with everolimus alone in a randomized phase II trial [27].

\section{$\underline{\text { Vaccines }}$}

As tumours progress they induce immune tolerance by loss of expression of tumour antigens and expression of checkpoint ligands leading to T-cell exhaustion. Viral vaccines have been designed to express pre-selected shared tumour antigens, which lead to activation of anti-tumour T-cells. The TroVax Renal Immunotherapy Survival phase III Trial evaluated standard of care alone or with a modified vaccinia Ankara encoding the tumour antigen 5T4 (MVA-5T4) for the treatment for mRCC [28]. Unfortunately, significant difference in overall survival was not observed, although, the magnitude of 5T4-specific antibody response and normal hematologic parameters (platelet, monocytes, haemoglobin) were associated with better survival.

Following on from phase 1 and 2 studies demonstrating safety and correlation between immunological response and clinical outcomes [29,30], IMPRINT was a phase 3 study of the IMA901 vaccine with 10 tumour antigens (9 binding HLA-A*02 and 1 binding HLA-DR) known to be overexpressed on RCC [31]. Uniquely, this trial investigated the combination of 
a vaccine with a VEGF inhibiting TKI. Vaccination with IMA901 plus GM-CSF and sunitinib were compared with sunitinib alone in $\mathrm{HLA}-\mathrm{A}^{*} 02$ positive mccRCC patients. The median OS was 33 months for the vaccine combination and was not reached for sunitinib. PFS was 15 months for both arms. As such, despite promising early phase data the phase 3 study did not meet its primary endpoint. Interestingly, the T-cell response in the phase 3 study was three times less than that of the phase 1/2 studies of IMA901 [32].

The phase 3 ADAPT trial (NCT01582672) is due to report and combines a customized patient-specific dendritic cell vaccine (AGS-003) with or without sunitinib. Previous preclinical studies that sunitinib suppressed myeloid derived suppressor cells (MDSCs) probably mediated by STAT3 inhibition, suggesting potential synergism with immunotherapy $[33,34]$. AGS-003 is composed of mature dendritic cells collected by leukapheresis, which is then electroporated with mRNA derived from patient's tumour and with CD40 ligand. There is great interest in the result of this combination study as the phase 2 study demonstrated a good median OS of 30 months for combined vaccine and sunitinib [35].

These collective results indicate no role for vaccines in mRCC currently, as stand alone or in combination with VEGF TKIs. However, there are no phase III study results combining vaccines with T-cell checkpoint inhibitors. There are issues over the cost of patient specific vaccine products and also over the potential for changes in trial comparator agents from TKIs to T-cell checkpoint inhibitors as first line therapy for mRCC when much anticipated first line checkpoint inhibitor trials readout soon [32].

T-cell checkpoint inhibitor combination of PD-1/PD-L1 and CTLA-4 inhibitors

PD-1/PD-L1 inhibitors lend themselves to combinations owing to their excellent therapeutic index, with grade $\geq 3$ toxicities in only $15-20 \%$ of patients. The CTLA- 4 receptor is essential for the function of CD4 T-regulator cells which are the key cells enabling self-tolerance.

Whereas, PD-1 is an inhibitory receptor expressed on T-cells which when bound to its ligand 
PD-L1 on tumour cells dampens the effect of CD8 T-cells and can cause exhausted T-cells. Figure 1 illustrates that CTLA-4 and PD-1/PD-L1 inhibitors work in different components of the cancer-immunity cycle, suggesting that this combination is non-redundant [36]. One of the first phase 3 trials in this arena was in advanced, previously untreated melanoma where combination CTLA-4 (ipilimumab) and PD-1 (nivolumab) inhibition resulted in an objective response rate (ORR) of $57.6 \%$ for combination therapy compared to $43.7 \%$ and $19 \%$ with nivolumab and ipilimumab monotherapies respectively [37]. Pre-clinical work had established that the potency of this combination therapy was likely due to the fact that the 2 agents work on difference cell types. Interestingly in both mouse and cell line models, this same effect was not seen with sequential therapy [38].

In mRCC, the phase I CHECKMATE 016 trial (NCT01472081) and expansion phase of that study assessed the combination of nivolumab (PD-1 inhibitor) and ipilimumab (CTLA-4 inhibitor) in the first (53\%) and second line (47\%) setting, using two different dosing regimens [39]. The ORR was 40\% for nivolumab $3 \mathrm{mg} / \mathrm{kg}$ plus ipilimumab $1 \mathrm{mg} / \mathrm{kg}$ (N3/1) and nivolumab $1 \mathrm{mg} / \mathrm{kg}$ plus ipilimumab $3 \mathrm{mg} / \mathrm{kg}$ (N1/3). In both groups 83\% had either CR, PR or SD. The N3I1 appeared more tolerable and yielded CR in 10\% patients, with many patients exhibiting durable responses [39]. However, the combination does produce a greater risk of toxicities with grade $\geq 3$ toxicities in approximately 40 to $60 \%$ of patients when examining patients across different malignancies. Nevertheless, these promising response data triggered a phase III U.S. Intergroup trial comparing first-line nivolumab combined with ipilimumab vs sunitinib (CHECKMATE 214; NCT02231749) [40].

\section{$\underline{\text { Immune and targeted drug combinations }}$}

When considering the cancer-immunity cycle (figure 1), various oncology drugs can interfere with potentially beneficial effects for the mRCC patient [36]. There is a strong biological rationale for combining immunotherapy with VEGF targeting agents (i.e. sunitinib, axitinib, 
bevacuzimab) as the latter agents can promote infiltration of T-cells into tumours and facilitate synergism with PD-1 or PD-L1 inhibitors.

The story in human studies is as follows. Phase 1 studies of nivolumab plus sunitinib or pazopanib in mRCC showed PFS rate at 24 weeks was $78 \%$ for sunitinib arm and $55 \%$ for the pazopanib arm [41]. However, there were 4 dose limiting toxicities (elevated ALT/AST [n=3], fatigue [n=1]) in 20 patients treated with pazopanib resulting in closure of this arm of the study. In contrast, a phase 1b study in the first line mRCC setting investigating the combination of avelumab and axitinib demonstrated feasibility and clinical benefit in all 6 patients studied, with partial response in 5 patients [37]. These results have provided a rationale to undertake a phase 3 study of avelumab combined with axitinib vs sunitinib [43].

An alternative combination of atezolizumab (PD-L1 inhibitor) and bevacizumab in previously untreated $\mathrm{mRCC}$ in a phase 1 study demonstrated partial responses in 4 of 10 patients, with an additional 4 patients having prolonged stable disease [44]. Historical ORR for atezolizumab and bevacizumab are $15 \%$ and $9 \%$ respectively [44]. In correlative work from this study, intra-tumoural $C D 8^{+} \mathrm{T}$ cells were found to increase following combination treatment, suggesting that the anti-VEGF and anti-PD-L1 combination improves antigenspecific T-cell migration [44]. Ongoing studies using this combination include: phase II trial (NCT01984242) of atezolizumab monotherapy vs atezolizumab plus bevacizumab vs sunitinib and a phase III trial of atezolizumab plus bevacizumab vs sunitinib (NCT02420821).The randomized phase II trial was recently reported and demonstrated no overall statistical differences in PFS, but patients with PD-L1 positive tumours demonstrated a trend for extension of PFS with the combination vs sunitinib ( $H R=0.65, p=0.095)$ [45].

\section{Evidence synthesis}


There is no evidence regarding the optimal sequencing of TKIs and immunotherapy. Indeed, despite the strategy of combining agents, a substantial proportion of patients will be ineligible for more toxic combinations and may warrant the serial delivery of single agents. The randomized phase 2 SUAVE trial (figure 2) will compare avelumab (PD-L1 inhibitor) followed by sunitinib on PD vs sunitinib followed by avelumab on PD, and evaluates biomarkers to guide future development and therapy. Given the difficulty of comparing the multitude of possible sequences of multiple lines of therapy, a definitive resolution to the conundrum is unlikely.

In other malignancies, expression of PD-L1 on cancer cells or infiltrating immune cells has been both prognostic and predictive. However, in RCC the expression of PD-L1 has been found to be highly variable. This is likely due to a mixture of factors: different antibodies, differing staining patterns (membrane vs cytoplasm), differing cut-offs for expression, whether cancer cell or immune cell expression is assessed [9]. In the $2^{\text {nd }}$ line phase 3 trials of PD-1 inhibitors undertaken to date expression of PD-L1 appeared prognostic for worse survival, but was not predictive. In combination studies with a VEGF TKI, PD-L1 status was inversely correlated with response [41].

Phase 2 studies where pre- and post-therapy tissue samples have been obtained may be the ideal setting to develop correlative biomarkers, both prognostic/predictive biomarkers and mechanism of resistance. Indeed, insights into early emerging pathways mediating resistance may assist with developing optimal combinations. Ball et al have undertaken a study comparing 4 exceptional responders to nivolumab with 3 patients with primary refractory disease [46]. The results demonstrated an increased CD8 lymphocyte infiltrate, number of somatic mutations, mutation associated neoantigens and expression of proinflammatory immune transcripts in exceptional responders compared to patients with primary refractory disease. As with all molecular studies there is a need for extensive independent validation before a biomarker has truly been developed [47]. 
Considering combination and sequential therapy with immunooncology agents there will be an increasing need to advance the development of predictive biomarkers in order to rationalize the multitude of potential combinations available for each patient. Molecular factors to select for efficacy and toxicity are not ready for routine use, but in the long term will hopefully make precision medicine possible. In the absence of validated predictive biomarkers, physicians should probably attempt to deliver as many lines of therapy as possible based on approved indications. Trials should be offered for every line of therapy since cure is unlikely with current therapy.

\section{Conclusions}

It remains to be established if combination of immunotherapy agents will provide a synergistic response in RCC as is the case in other diseases such as melanoma. If they do, then it may be that combinatorial therapies become the standard of first line care. If not and they are merely additive then the sequencing of agents will need to be more closely established as this may alter efficacy but reduce toxicity. Nevertheless, sequencing of single agents may continue to play a major role in patients unable to tolerate combination therapy due to comorbidities or suboptimal performance status. 
Acknowledgements

There was no funding for this research. 


\section{References}

[1] Vogelzang NJ, Priest ER, Borden L. Spontaneous regression of histologically proved pulmonary metastases from renal cell carcinoma: a case with 5-year followup. J Urol 1992;148:1247-8.

[2] Papac RJ. Spontaneous regression of cancer. Cancer Treat Rev 1996;22:395-423.

[3] Lokich J. Spontaneous regression of metastatic renal cancer. Case report and literature review. Am J Clin Oncol 1997;20:416-8.

[4] Raman R, Vaena D. Immunotherapy in Metastatic Renal Cell Carcinoma: A Comprehensive Review. BioMed Res Int 2015;2015:367354. doi:10.1155/2015/367354.

[5] Motzer RJ. Perspective: What next for treatment? Nature 2016;537:S111. doi:10.1038/537S111a.

[6] Motzer RJ, Hutson TE, Cella D, Reeves J, Hawkins R, Guo J, et al. Pazopanib versus sunitinib in metastatic renal-cell carcinoma. N Engl J Med 2013;369:722-31. doi:10.1056/NEJMoa1303989.

[7] Albiges L, Oudard S, Negrier S, Caty A, Gravis G, Joly F, et al. Complete remission with tyrosine kinase inhibitors in renal cell carcinoma. J Clin Oncol Off J Am Soc Clin Oncol 2012;30:482-7. doi:10.1200/JCO.2011.37.2516.

[8] Motzer RJ, Escudier B, McDermott DF, George S, Hammers HJ, Srinivas S, et al. Nivolumab versus Everolimus in Advanced Renal-Cell Carcinoma. N Engl J Med 2015;373:1803-13. doi:10.1056/NEJMoa1510665.

[9] Harshman LC, Drake CG, Choueiri TK. PD-1 blockade in renal cell carcinoma: to equilibrium and beyond. Cancer Immunol Res 2014;2:1132-41. doi:10.1158/23266066.CIR-14-0193.

[10] Pardoll D, Drake C. Immunotherapy earns its spot in the ranks of cancer therapy. J Exp Med 2012;209:201-9. doi:10.1084/jem.20112275.

[11] Ball MW, Allaf ME, Drake CG. Recent advances in immunotherapy for kidney cancer. Discov Med 2016;21:305-13.

[12] Vanneman M, Dranoff G. Combining immunotherapy and targeted therapies in cancer treatment. Nat Rev Cancer 2012;12:237-51. doi:10.1038/nrc3237.

[13] McDermott DF, Regan MM, Clark JI, Flaherty LE, Weiss GR, Logan TF, et al. Randomized phase III trial of high-dose interleukin-2 versus subcutaneous interleukin-2 and interferon in patients with metastatic renal cell carcinoma. J Clin Oncol Off J Am Soc Clin Oncol 2005;23:133-41. doi:10.1200/JCO.2005.03.206.

[14] Fisher RI, Rosenberg SA, Fyfe G. Long-term survival update for high-dose recombinant interleukin-2 in patients with renal cell carcinoma. Cancer J Sci Am 2000;6 Suppl 1:S55-57.

[15] Bui MHT, Seligson D, Han K, Pantuck AJ, Dorey FJ, Huang Y, et al. Carbonic anhydrase IX is an independent predictor of survival in advanced renal clear cell carcinoma: implications for prognosis and therapy. Clin Cancer Res Off J Am Assoc Cancer Res 2003;9:802-11.

[16] Atkins M, Regan M, McDermott D, Mier J, Stanbridge E, Youmans A, et al. Carbonic anhydrase IX expression predicts outcome of interleukin 2 therapy for renal cancer. Clin Cancer Res Off J Am Assoc Cancer Res 2005;11:3714-21. doi:10.1158/10780432.CCR-04-2019.

[17] McDermott DF, Cheng S-C, Signoretti S, Margolin KA, Clark JI, Sosman JA, et al. The high-dose aldesleukin "select" trial: a trial to prospectively validate predictive models of response to treatment in patients with metastatic renal cell carcinoma. Clin Cancer Res Off J Am Assoc Cancer Res 2015;21:561-8. doi:10.1158/1078-0432.CCR-14-1520.

[18] Upton MP, Parker RA, Youmans A, McDermott DF, Atkins MB. Histologic predictors of renal cell carcinoma response to interleukin-2-based therapy. J Immunother Hagerstown Md 1997 2005;28:488-95.

[19] Erbe AK, Wang W, Goldberg J, Gallenberger M, Kim K, Carmichael L, et al. FCGR Polymorphisms Influence Response to IL-2 in Metastatic Renal Cell Carcinoma. Clin Cancer Res Off J Am Assoc Cancer Res 2016. doi:10.1158/1078-0432.CCR-16-1874. 
[20] Rini BI, Halabi S, Rosenberg JE, Stadler WM, Vaena DA, Ou S-S, et al. Bevacizumab plus interferon alfa compared with interferon alfa monotherapy in patients with metastatic renal cell carcinoma: CALGB 90206. J Clin Oncol Off J Am Soc Clin Oncol 2008;26:5422-8. doi:10.1200/JCO.2008.16.9847.

[21] Escudier B, Pluzanska A, Koralewski P, Ravaud A, Bracarda S, Szczylik C, et al. Bevacizumab plus interferon alfa-2a for treatment of metastatic renal cell carcinoma: a randomised, double-blind phase III trial. Lancet Lond Engl 2007;370:2103-11. doi:10.1016/S0140-6736(07)61904-7.

[22] Dandamudi UB, Ghebremichael M, Sosman JA, Clark JI, McDermott DF, Atkins MB, et al. A phase II study of bevacizumab and high-dose interleukin-2 in patients with metastatic renal cell carcinoma: a Cytokine Working Group (CWG) study. J Immunother Hagerstown Md 1997 2013;36:490-5. doi:10.1097/CJl.0000000000000003.

[23] Motzer RJ, Hudes G, Wilding G, Schwartz LH, Hariharan S, Kempin S, et al. Phase I trial of sunitinib malate plus interferon-alpha for patients with metastatic renal cell carcinoma. Clin Genitourin Cancer 2009;7:28-33. doi:10.3816/CGC.2009.n.005.

[24] Patel PH, Senico PL, Curiel RE, Motzer RJ. Phase I study combining treatment with temsirolimus and sunitinib malate in patients with advanced renal cell carcinoma. Clin Genitourin Cancer 2009;7:24-7. doi:10.3816/CGC.2009.n.004.

[25] Rini BI, Bellmunt J, Clancy J, Wang K, Niethammer AG, Hariharan S, et al. Randomized phase III trial of temsirolimus and bevacizumab versus interferon alfa and bevacizumab in metastatic renal cell carcinoma: INTORACT trial. J Clin Oncol Off J Am Soc Clin Oncol 2014;32:752-9. doi:10.1200/JCO.2013.50.5305.

[26] Ravaud A, Barrios CH, Alekseev B, Tay M-H, Agarwala SS, Yalcin S, et al. RECORD2: phase II randomized study of everolimus and bevacizumab versus interferon $\alpha-2 a$ and bevacizumab as first-line therapy in patients with metastatic renal cell carcinoma. Ann Oncol Off J Eur Soc Med Oncol 2015;26:1378-84. doi:10.1093/annonc/mdv170.

[27] Motzer RJ, Hutson TE, Glen H, Michaelson MD, Molina A, Eisen T, et al. Lenvatinib, everolimus, and the combination in patients with metastatic renal cell carcinoma: a randomised, phase 2, open-label, multicentre trial. Lancet Oncol 2015;16:1473-82. doi:10.1016/S1470-2045(15)00290-9.

[28] Amato RJ, Hawkins RE, Kaufman HL, Thompson JA, Tomczak P, Szczylik C, et al. Vaccination of metastatic renal cancer patients with MVA-5T4: a randomized, doubleblind, placebo-controlled phase III study. Clin Cancer Res Off J Am Assoc Cancer Res 2010;16:5539-47. doi:10.1158/1078-0432.CCR-10-2082.

[29] Kirner A, Mayer-Mokler A, Reinhardt C. IMA901: a multi-peptide cancer vaccine for treatment of renal cell cancer. Hum Vaccines Immunother 2014;10:3179-89. doi:10.4161/21645515.2014.983857.

[30] Walter S, Weinschenk T, Stenzl A, Zdrojowy R, Pluzanska A, Szczylik C, et al. Multipeptide immune response to cancer vaccine IMA901 after single-dose cyclophosphamide associates with longer patient survival. Nat Med 2012;18:1254-61. doi:10.1038/nm.2883.

[31] Rini BI, Stenzl A, Zdrojowy R, Kogan M, Shkolnik M, Oudard S, et al. IMA901, a multipeptide cancer vaccine, plus sunitinib versus sunitinib alone, as first-line therapy for advanced or metastatic renal cell carcinoma (IMPRINT): a multicentre, open-label, randomised, controlled, phase 3 trial. Lancet Oncol 2016;17:1599-611. doi:10.1016/S1470-2045(16)30408-9.

[32] Pal SK, Agarwal N, Dizman N, Sonpavde G. Vaccine therapy in renal cell carcinoma: attempting to leap over a rising bar. Lancet Oncol 2016;17:1477-8. doi:10.1016/S14702045(16)30493-4.

[33] Hipp MM, Hilf N, Walter S, Werth D, Brauer KM, Radsak MP, et al. Sorafenib, but not sunitinib, affects function of dendritic cells and induction of primary immune responses. Blood 2008;111:5610-20. doi:10.1182/blood-2007-02-075945. 
[34] Xin H, Zhang C, Herrmann A, Du Y, Figlin R, Yu H. Sunitinib inhibition of Stat3 induces renal cell carcinoma tumor cell apoptosis and reduces immunosuppressive cells.

Cancer Res 2009;69:2506-13. doi:10.1158/0008-5472.CAN-08-4323.

[35] Amin A, Dudek AZ, Logan TF, Lance RS, Holzbeierlein JM, Knox JJ, et al. Survival with AGS-003, an autologous dendritic cell-based immunotherapy, in combination with sunitinib in unfavorable risk patients with advanced renal cell carcinoma (RCC): Phase 2 study results. J Immunother Cancer 2015;3:14. doi:10.1186/s40425-015-0055-3.

[36] Chen DS, Mellman I. Oncology Meets Immunology: The Cancer-Immunity Cycle. Immunity 2013;39:1-10. doi:10.1016/j.immuni.2013.07.012.

[37] Larkin J, Chiarion-Sileni V, Gonzalez R, Grob JJ, Cowey CL, Lao CD, et al. Combined Nivolumab and Ipilimumab or Monotherapy in Untreated Melanoma. N Engl J Med 2015;373:23-34. doi:10.1056/NEJMoa1504030.

[38] Selby MJ, Engelhardt JJ, Johnston RJ, Lu L-S, Han M, Thudium K, et al. Preclinical Development of Ipilimumab and Nivolumab Combination Immunotherapy: Mouse Tumor Models, In Vitro Functional Studies, and Cynomolgus Macaque Toxicology. PLOS ONE 2016;11:e0161779. doi:10.1371/journal.pone.0161779.

[39] Hammers HJ, Plimack ER, Infante JR, Rini BI, McDermott DF, Ernstoff M, et al. Expanded cohort results from CheckMate 016: A phase I study of nivolumab in combination with ipilimumab in metastatic renal cell carcinoma (mRCC). J Clin Oncol 2015;33.

[40] Hammers HJ, Plimack ER, Sternberg C, McDermott DF, Larkin JMG, Ravaud A, et al. CheckMate 214: A phase III, randomized, open-label study of nivolumab combined with ipilimumab versus sunitinib monotherapy in patients with previously untreated metastatic renal cell carcinoma. J Clin Oncol 2015;33.

[41] Amin A, Plimack ER, Infante JR, Ernstoff MS, Rini BI, McDermott DF, et al. Nivolumab (anti-PD-1; BMS-936558, ONO-4538) in combination with sunitinib or pazopanib in patients (pts) with metastatic renal cell carcinoma (mRCC). J Clin Oncol 2014;32:5s.

[42] Larkin J, Rini BI, Nathan P, Thistlethwaite F, Gordon M, Martgnoni M, et al. Phase 1b dose-finding study of avelumab (anti-PD-L1) + axitinib in treatment-naïve patients with advanced renal cell carcinoma. Ann Oncol 2016;27:775PD. doi:10.1093/annonc/mdw373.03.

[43] Motzer RJ, Choueiri T, Larkin J, Albiges L, Haanen JB, Schmidinger M, et al. Phase 3 study of avelumab in combination with axitinib versus sunitinib as first-line treatment for patients with advanced renal cell carcinoma (aRCC). Ann Oncol 2016;27:844TiP. doi:10.1093/annonc/mdw373.71.

[44] Wallin JJ, Bendell JC, Funke R, Sznol M, Korski K, Jones S, et al. Atezolizumab in combination with bevacizumab enhances antigen-specific T-cell migration in metastatic renal cell carcinoma. Nat Commun 2016;7:12624. doi:10.1038/ncomms12624.

[45] McDermott DF, Atkins MB, Motzer RJ, Rini BI, Escudier BJ, Fong L, et al. A phase II study of atezolizumab (atezo) with or without bevacizumab (bev) versus sunitinib (sun) in untreated metastatic renal cell carcinoma (mRCC) patients (pts). J Clin Oncol 2017;35.

[46] Ball MW, Johnson MH, Gorin MA, Rodriguez M, Diaz LA, Haffner M, et al. Clinical, pathologic, and genomic profiles of exceptional responders to anti-PD1 therapy in renal cell carcinoma. J Clin Oncol 2016;34.

[47] Diamandis EP. Cancer biomarkers: can we turn recent failures into success? J Natl Cancer Inst 2010;102:1462-7. doi:10.1093/jnci/djq306. 
Table 1. Ongoing randomized phase III combination T-cell checkpoint inhibitor trials in metastatic ccRCC

\begin{tabular}{|l|l|l|l|}
\hline Therapeutic target & Line & Control arm & Experimental arm(s) \\
\hline PD-1 and CTLA-4 & First & Sunitinib & $\begin{array}{l}\text { Nivolumab + Ipilimumab x 4 } \\
\text { Nivolumab }\end{array}$ \\
\hline PD-1 and VEGF & First & Sunitinib & Bevacizumab + Atezolizumab \\
\hline PD-L1 and VEGF & First & Sunitinib & Axitinib + Avelumab \\
\hline $\begin{array}{l}\text { PD-L1 and VEGF } \\
\text { VEGF/FGF and (PD-1 or } \\
\text { mTOR) }\end{array}$ & First & Sunitinib & Axitinib + Pembrolizumab \\
\hline $\begin{array}{l}\text { Vaccine (Dendritic cell- } \\
\text { based vaccine }+ \\
\text { autologous tumour cell } \\
\text { mRNA }+ \text { CD40 ligand) }\end{array}$ & First & Sunitinib & $\begin{array}{l}\text { Lenvatinib + Pembrolizumab OR } \\
\text { Lenvatinib + Everolimus }\end{array}$ \\
\hline
\end{tabular}


Figure 1. The cancer-immunity cycle and effect of therapies

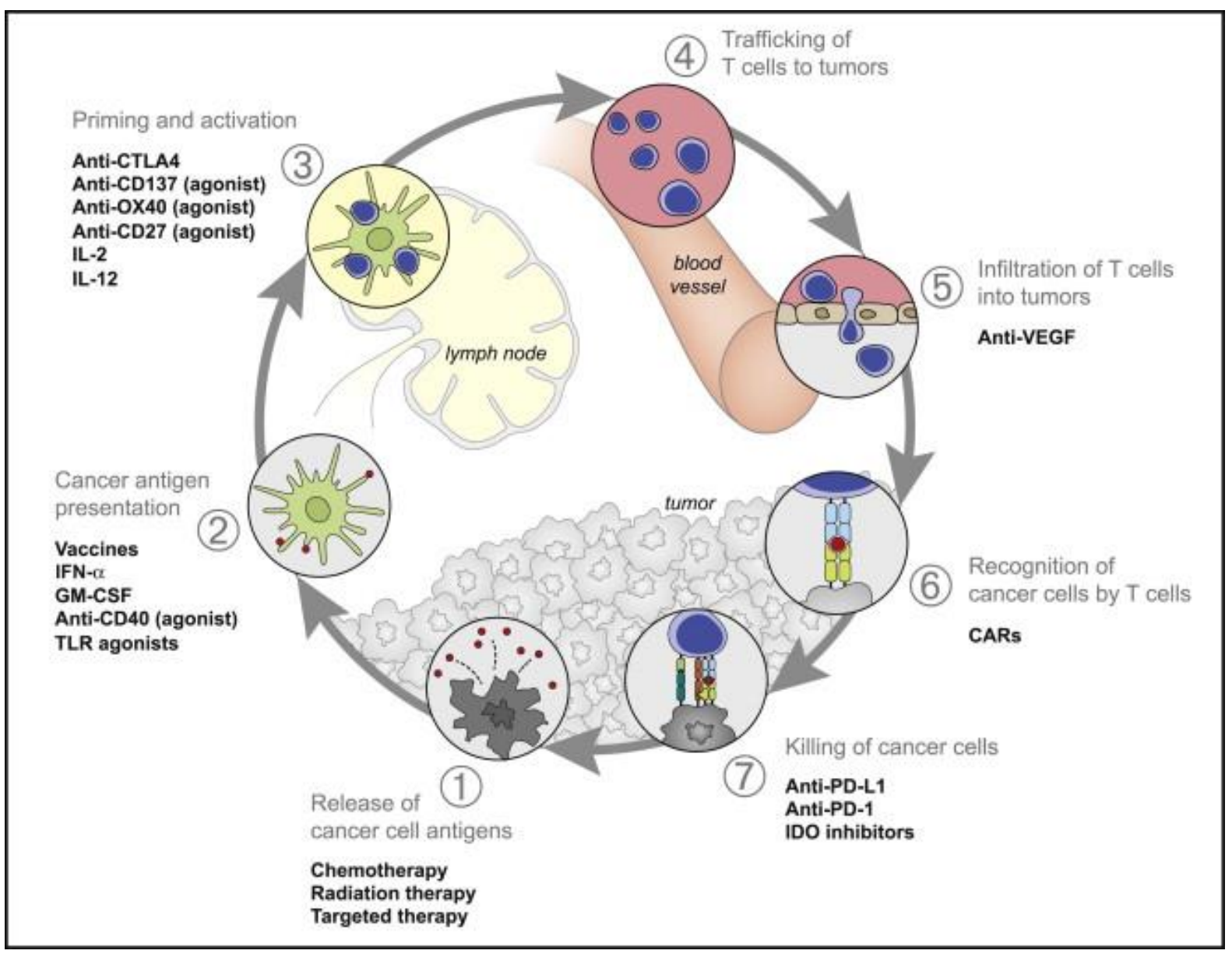

The process of generation of immunity is cyclical and leads to factors which stimulate the immune system to produce a T-cell response. Conversely, there are also immune-inhibiting factors which regulate immunity. Beneath each of the 7 steps of the cancer-immunity cycle are examples of therapies that may interplay at that point [36]. Abbreviations: GM-CSF, granulocyte macrophage colony-stimulating factor; CARs, chimeric antigen receptors (taken from [36] with permission). 
Figure 2. Design of SUAVE trial
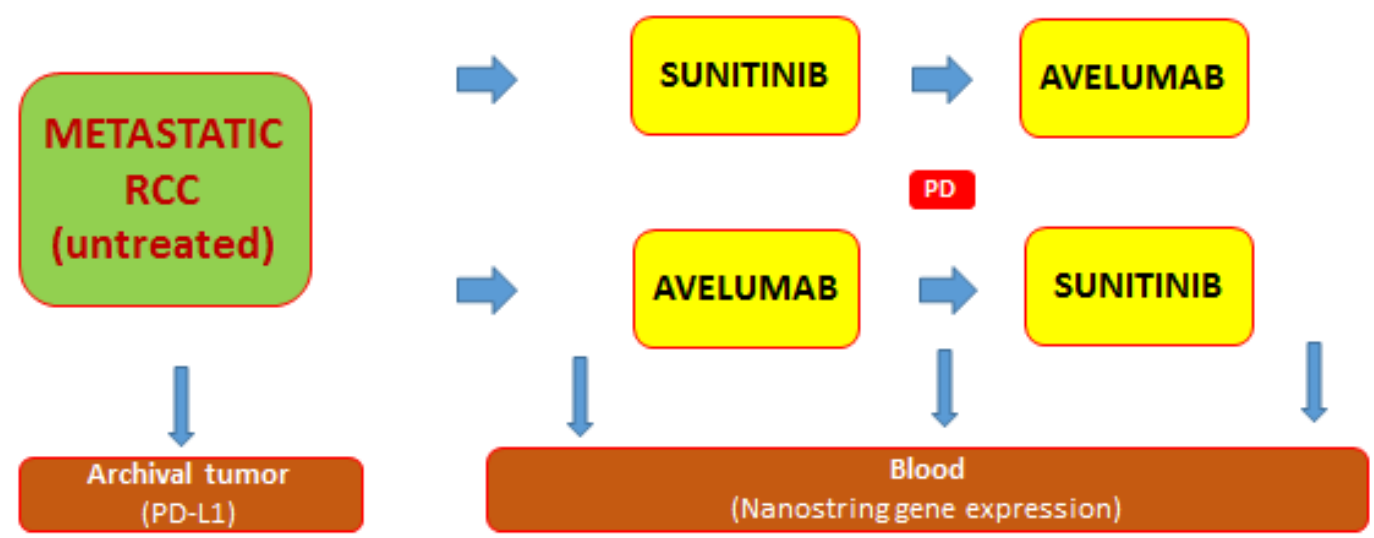